\title{
Verdad y justicia en Olavarría: el caso del recital de Indio Solari
}

\section{Truth and justice in Olavarría: the case of Indio Solari's recital}

\author{
Brenda Mirella Bahl brendambahl@gmail.com \\ http://orcid.org/0000-0002-6273-8356 \\ Universidad Nacional del Centro de la Provincia de Buenos Aires, Argentina
}

Resumen

Durante un recital de rock masivo llevado a cabo por el artista Indio Solari en la ciudad de Olavarría en marzo de 2017, dos personas fallecieron. Este suceso llevó a numerosos actores sociales a presentar el evento como una tragedia, y a discutir en la escena pública las responsabilidades al respecto. En este contexto, se conformó la comisión ¿qué pasó en Olavarría? Por la Verdad y Justicia (en adelante CVJ), integrada por vecinas y vecinos de la ciudad, estudiantes universitarios y militantes políticos. La CVJ presentó como su principal objetivo denunciar públicamente al gobierno municipal, provincial y nacional a través de una movilización en la plaza central de la ciudad. La intención de este artículo será, desde un enfoque etnográfico, dilucidar cómo las categorías nativas verdad y justicia producidas por la 
CVJ, operaron como un tejido discursivo legítimo para la acreditación en la arena pública del carácter trágico de la situación social analizada. Se mostrará cómo estas nociones vernáculas aparecieron como síntesis sociales de una trama de significaciones más amplia vinculada al repertorio de los organismos y militantes por los Derechos Humanos, y que resultaron fundamentales como estrategia para la búsqueda de apoyo político de actores sociales diversos.

Palabras Clave: antropología política; tragedia; verdad y justicia; tejido discursivo.

\section{Abstract}

During a massive rock recital conducted by the Indian artist Solari in the city of Olavarría in March 2017, two people died. This event led numerous social actors to present the event as a tragedy, and to discuss on the public scene the responsibilities in this regard. In this context, the commission was formed, what happened in Olavarría? For the Truth and Justice (hereinafter CVJ), composed of neighbors of the city, university students and political militants. The CVJ presented as its main objective publicly denouncing the municipal, provincial and national government through a mobilization in the central square of the city. The intention of this article will be, from an ethnographic approach, to elucidate how the native truth and justice categories produced by the CVJ, operated as a legitimate discursive fabric for the accreditation in the public arena of the tragic character of the analyzed social situation. It will be shown how these vernacular notions appeared as social syntheses of a broader plot of meanings linked to the repertoire of human rights organizations and activists, and which were fundamental as a strategy for seeking political support from diverse social actors.

Keywords: political anthropology; tragedy; truth and justice; discursive tissue. 


\section{Introducción}

El día 11 de marzo de 2017 en la ciudad de Olavarría ${ }^{1}$ el artista Indio Solari ${ }^{2}$ junto a su banda los fundamentalistas del aire acondicionado, brindó un recital de rock masivo ${ }^{3}$ en el marco del cual dos personas murieron. Esta situación llevó a numerosos actores sociales a delinear la situación como trágica, y a movilizar diversas estrategias para presentar y disputar en la arena pública esta versión del caso. En este contexto, se conformó la Comisión ¿Qué pasó en Olavarría? Por la Verdad y Justicia (en adelante CVJ), integrada por vecinas y vecinos de la ciudad, estudiantes universitarios y militantes políticos. La CVJ presentó como su principal objetivo denunciar públicamente al gobierno municipal, provincial y nacional a través de una movilización en la plaza central de la ciudad.

Las consideraciones aquí presentadas se desprenden de una investigación de grado para la obtención del título de Licenciatura en Antropología con orientación social de la Universidad Nacional del Centro de la Provincia de Buenos Aires. La misma, orientó sus esfuerzos a la interpretación de la producción social del caso del recital del Indio Solari en Olavarría, particularmente al análisis de las formas sociales que asumieron las discusiones y debates en la contienda bajo la delimitación analítica de <<controversia pública >>.

La reconstrucción etnográfica y procesual de la situación social (Gluckman, 1958) estudiada mostró que la controversia resultó de un proceso colectivo de definición, donde participaron una gran heterogeneidad de actores sociales: desde vecinas y vecinos de la ciudad, funcionarios y funcionarias municipales, ministros provinciales y nacionales, agentes del sector judicial, expertos en música de rock y la psicología, personas de la comunidad ricotera, militantes políticos, entre otros. En este artículo se focalizará el análisis en un actor en particular: la Comisión por la Verdad y la Justicia en Olavarría (CVJ). La CVJ nucleó una serie de actores locales (militantes políticos de Partido Obrero (en adelante, $\mathrm{PO}$ ), vecinos y vecinas de la ciudad y estudiantes universitarios) que colaboraron en la asistencia de quienes habían

1 Olavarría es la ciudad de cabecera del partido que lleva su mismo nombre, ubicada entre la Ruta Nacional № 226 y la Ruta Provincial №51. Según los resultados del último Censo Nacional (2010) consta de una población de 111.708 habitantes. Se constituyó a partir de un entramado industrial que estuvo históricamente ligado a la minería y a la producción de materiales de construcción, especialmente el cemento. En la actualidad en este entramado se incluye la producción agropecuaria, lo que hizo que la ciudad se consolidara como uno de los principales distritos ganaderos de la Provincia de Buenos Aires.

${ }^{2}$ Se utilizará entrecomillado para referir a categorías y conceptos teóricos y cursiva para señalar categorías que forman parte del discurso nativo reconstruido a partir del trabajo de campo.

${ }^{3}$ Según informes oficiales publicados en el periódico local El Popular del día 11 de marzo de 2018, se estima un número aproximado de 225 mil a 245 mil personas asistentes. 
quedado varados en la ciudad una vez finalizado el recital y con la imposibilidad de comunicarse con sus familiares.

Unos días después del evento y frente a un contexto de incertidumbre sobre la cantidad de personas perdidas y fallecidas, la CVJ convoca a una movilización en la plaza central de la ciudad. Alli se expuso una gran preocupación por mostrar que se trataba de un grupo integrado por sectores sociales diversos, pero que compartían un objetivo en común: denunciar a funcionarios políticos de diversas escalas por las muertes producidas en el recital y por sucesos posteriores que la CVJ calificó con nociones como desastre, descontrol, caos y negligencia.

El objetivo de este artículo será dilucidar cómo las categorías nativas verdad y justicia, producidas y presentadas por la CVJ, operaron como un tejido discursivo legítimo para acreditación en la arena pública (Gusfield, 2014; Cefaï, 2012) del carácter trágico de la situación social analizada, pero también como dispositivos de denuncia y reclamo al Estado. A su vez, y a la luz de los aportes de Da Silva Catela (2008) y Fonseca y Cardarello (2004) mostraremos cómo estas nociones vernáculas aparecieron como síntesis sociales de una trama de significaciones más amplia vinculada al repertorio de los organismos y militantes por los Derechos Humanos, y que resultaron fundamentales como estrategia para la búsqueda de apoyo político de actores sociales diversos.

En cuanto a la metodología, la investigación se desarrolló a partir de un enfoque etnográfico, cuya principal característica es la reconstrucción del punto de vista nativo y los significados locales envueltos en los procesos sociales estudiados, a partir de la documentación de lo no documentado (Rockwell, 2009). Las principales técnicas desarrolladas para la reconstrucción de datos de campo fueron la observación participante y entrevistas etnográficas.

A su vez, y atendiendo a la característica del caso en tanto evento temporalmente acotado, se recuperaron algunos aportes metodológicos de la Escuela de Manchester para el estudio dinámico y procesual de los conflictos: el <<análisis situacional>> desarrollado por Gluckman (1958). El autor define por <<situación social >> a aquellos momentos donde se desarrollan incidentes dramáticos, o más bien <<conflictos vividos en el marco de relaciones sociales tensas e inestables>> (Guizardi, 2012, p. 17). Es tarea del antropólogo o la antropóloga conectar los acontecimientos relevados, por lo que el $<<$ análisis situacional $>>$ requiere de un seguimiento de las prácticas de los actores, así como de la comprensión de los contextos en los que éstas se inscriben. Así, en esta investigación en particular, el trabajo de campo estuvo centrado en el registro etnográfico de una serie de eventos públicos (reuniones entre vecinos y el gobierno municipal, sesiones político-administrativas, movilizaciones públicas, etcétera), para 
mostrar la complejidad de sentidos creados y recreados en torno al tema, así como también de las dinámicas por las cuales las personas ocuparon y disputaron mutables y móviles "lugares". El artículo está organizado en partes: en la primera, se describe de forma general el caso del recital del Indio Solari en Olavarría; en la segunda, se reconstruye la movilización de la CVJ y se explican los diversos sentidos vinculados a la no politización de las demandas; en la tercera, se analiza qué lugar ocuparon las categorías de verdad y justicia para la acreditación de la situación como trágica, así como de su inscripción a un contexto más amplio vinculado al repertorio que diversos organismos, organizaciones y militantes por los Derechos Humanos construyeron para denunciar el terrorismo de Estado en Argentina durante la última dictadura militar; por último, se presentan las consideraciones finales.

\section{Una breve contextualización del caso}

El caso toma relevancia pública en noviembre del año 2016, cuando el intendente del partido de Olavarría anuncia desde su cuenta personal de Twitter que Indio Solari llevaría a cabo un recital en la ciudad homónima. A partir de allí, el tema comienza a ser parte de la agenda pública/mediática, tanto en los medios de comunicación locales como nacionales.

Ahora bien, durante el mes de febrero de 2017, fueron personas vecinas de los barrios que linderan el predio donde se llevó a cabo el show los primeros actores sociales que comenzaron a movilizar recursos para presentar públicamente sus disconformidades con respecto a la realización del recital en la ciudad. A través de diferentes estrategias (protestas en avenidas de los barrios, notas, entrevistas y cartas en periódicos locales), las vecinas y los vecinos exigieron que sea el intendente municipal quien debiera hacerse cargo de sus reclamos y cuestionamientos. El Departamento Ejecutivo Municipal (a partir de ahora DEM), liderado por el jefe comunal, decidió atender a las demandas de las y los vecinos a través de diversas acciones que tenían el objetivo de informar acerca del recital, e intentando instalar entre la comunidad olavarriense de que se trataría de un evento con un gran beneficio económico para la ciudad (febrero de 2017). Así, durante estas primeras instancias de la controversia, fueron configurándose en la escena pública dos posturas respecto a las expectativas que el recital generaba: por un lado, su caracterización como un evento de una gran oportunidad para la ciudad, defendida y expuesta particularmente por el DEM; por otro, las ideas vinculadas a que podría resultar una catástrofe, presentada por vecinas y vecinos de la ciudad.

Ya desde algunos días previos al recital, la ciudad transformó completamente su rutina y aspecto habitual. Una enorme cantidad de personas comenzaron a ocupar diferentes espacios urbanos creando un marco extraordinario en donde el recital marcaba toda la escena. En los 
alrededores del predio se instalaron numerosos puestos de ventas de bebida, comida y diferentes mercancías alusivas a la banda y el evento, por medio de los cuales las personas se encontraban, desplazaban y celebraban las circunstancias. Debido a que una gran cantidad de personas comenzaron a llegar para asistir al evento, sus vehículos de transporte (colectivos, combis) no pudieron ingresar a la ciudad y debieron estacionar en las banquinas de las rutas de ingreso. Esto tuvo como consecuencia que muchos asistentes caminaran varios kilómetros desde las rutas de acceso al predio donde se realizó el espectáculo. Esto último se tradujo en un flujo miles de personas en las avenidas principales.

Ahora bien, el recital debe ser entendido como un hito en el marco de la contienda, una situación que marcó un antes y un después, ya que el foco de las discusiones que diseñaron la controversia se redefinió a través de un proceso social vinculado con la muerte de dos personas. En el centro de las acaloradas discusiones ya no se ponía en cuestión si debía realizarse o no, sino bajo qué condiciones se llevaría a cabo. Aquí, la expresión lo que debía ser una fiesta, se convirtió en una tragedia se generalizó entre diversos actores sociales, entendiendo que la noción de tragedia abrigó una serie de circunstancias consideradas como indeseadas y que fueron definidas a posteriori de aquellos hechos desafortunados.

Cabe destacar que, una vez finalizado el show y por circunstancias diversas, miles de personas quedaron varadas en la ciudad sin posibilidad de comunicarse con sus familias o con medios de transporte para regresar a sus hogares. Frente a esta situación, vecinas y vecinos pedían a través de las redes sociales colaborar con la gente perdida brindando abrigo, comida, carga para celulares y liberando la conexión Wi-Fi de sus hogares. En este contexto, estudiantes y profesores/as de las facultades de medicina e ingeniería ofrecían la casa de estudios como refugio hasta que se pudiera aquellas personas encontraran la forma de regresar a sus hogares. Muchas de las personas que realizaron acciones de asistencia a las personas perdidas, conformaron luego la CVJ.

El día 21 de marzo se realizó una sesión extraordinaria del Honorable Concejo Deliberante (a partir de ahora, HCD). Si bien en un primer momento el cuerpo legislativo estuvo de acuerdo en llevar a cabo el recital en la ciudad, en esta fase de la situación se encontró ocupando otro espacio: exigiéndole al Poder Ejecutivo explicaciones en torno a lo sucedido. Durante la mencionada sesión se presenta un pedido de proceso de interpelación por parte de uno de los bloques opositores para que el jefe comunal <<concurra a brindar información sobre los antecedentes, actos y hechos de público conocimiento ocurridos en el último recital del Indio Solari>> (expediente HCD № 016/17). Luego de casi dos horas de sesión, el pedido fue aprobado por unanimidad y se llevó a cabo el día 27 de marzo en uno de los salones del edificio municipal. En el expediente podían observarse un total de 324 preguntas formuladas 
por las y los concejales de los diversos bloques políticos que integran el HCD. El proceso finalmente se llevó a cabo en un salón municipal, frente a los ojos de más de 200 vecinas y vecinos y numerosos medios de comunicación (locales y nacionales). Duró un total de cinco horas y media, con cuartos intermedios cada una hora. Sin ahondar en demasiados detalles, los cuestionamientos realizados por las y los concejales se vincularon, principalmente, a cuestiones que atañen a las funciones del DEM y en ningún momento se atribuyó la responsabilidad de las muertes al intendente (como sí lo creía la CVJ).

Este caso tuvo una serie de consecuencias administrativas y políticas: por un lado, lo expuesto en la interpelación fue tratado en lo que en términos nativos se denominó comisión, integrada por concejalas y concejales de todos los sectores y aprobada por el mismo HCD. La comisión tuvo la tarea de redactar un informe que sería derivado al ámbito judicial (para que se incorpore a la causa que tramita el caso) y a la Defensoría del Pueblo de la Provincia de Buenos Aires, el cual fue presentado y aprobado en una sesión del órgano legislativo el día 5 de octubre de 2017. Por otra parte, la conformación del DEM fue modificada debido a la renuncia del Jefe de Gabinete (persona que, además de tener una amplia trayectoria política en la ciudad, se encontraba personalmente cercano al intendente municipal) como consecuencia del desmanejo político del recital. Días posteriores a esa renuncia, el intendente municipal comunicó que el encargado de la comunicación oficial del municipio, la persona que dirigía el Área de Habilitaciones y el Secretario de Desarrollo Económico serían reemplazados.

\section{La movilización de la CVJ y la no politización de la demanda}

Como se ha expuesto en el apartado anterior, una vez que el show acabó, miles de personas quedaron varadas en la ciudad y sin posibilidad de establecer comunicación con sus familias debido a que las líneas de celulares se encontraron saturadas. Así, durante las primeras horas de la madrugada del domingo y en el resto del día, el escenario de la ciudad podría ser descripto como una marea de gente desorientada, buscando los lugares donde dejaron sus transportes, yendo y viniendo de los distintos centros de salud para consultar si allí se encontraban sus familiares, amigos o amigas. Frente a este ambiente de incertidumbre y preocupación, numerosos vecinos y vecinas comenzaron a realizar acciones de asistencia, brindando agua o comida, permitiendo el paso de personas a los sanitarios de sus hogares, ofreciendo carga de celulares, liberando sus redes Wi-Fi, entre otras.

Algunos de estos actores, decidieron nuclearse luego en un espacio que designaron bajo la denominación de comisión (la CVJ), cuyo principal objetivo fue denunciar una serie de situaciones que consideraron indebidas y como grupo que denuncia y reclama al mismo 
Estado. Como se menciona anteriormente, la misma estuvo nucleada por personas que se autodefinían como vecinas y vecinos de la ciudad, estudiantes universitarios y militantes políticos del PO.

Ahora bien, es a través de la distribución de un flyer digital que circuló en una red social, la CVJ convocó a una movilización para el día 17 de marzo. Las personas que asistieron a la convocatoria decidieron instalarse en una esquina de la plaza central de la ciudad, la cual forma parte de una calle peatonal que se encuentra frente al llamado palacio municipal. Esto no es casual, ya que allí desarrollan sus actividades el intendente municipal y el resto de los funcionarios que pertenecen al DEM, actores que fueron fuertemente interpelados por la CVJ. Una vez allí, las y los integrantes de la CVJ dispusieron de un equipo musical y un micrófono de pie. Momentos antes de que una de sus integrantes llevase a cabo la lectura del comunicado, decidieron reproducir música del grupo Callejeros. Toda la performance que llevó a cabo la CVJ se realizó ahí, apuntando al palacio municipal y actuando como si allí estuvieran los actores a quienes eran dirigidas las acusaciones.

Como señalan Gusfield (2014) y Cefaï (2014) el componente escénico - es decir, la dimensión dramática - tiene una profunda importancia en la interpretación de los conflictos y controversias públicas. Bajo esta designación nativa de movilización, la CVJ usó la plaza central de la ciudad como escenario público para presentar sus reclamos, denuncias y argumentos. Siguiendo a Pita (2009), se comprendió entonces a la movilización como una <<forma social de protesta〉>, es decir, como formas sociales de manifestar el disenso (contra el DEM, por ejemplo), a la par que funciona como una herramienta para construir nuevas legitimidades (la CVJ como grupo que reclama en la arena pública).

En la puesta en escena de la CVJ no existía simplemente una preocupación por ser vistos (es decir, visibilizar sus reclamos), sino también por cómo y por quiénes querían ser vistos. Sus esfuerzos estuvieron centrados, principalmente, en instalar en la arena pública la responsabilidad política del DEM y otros actores sociales de la arena política provincial y nacional sobre lo sucedido durante el recital. Los argumentos presentados por la CVJ que reforzaron la acusación de responsabilidad se vincularon con la inexistencia de un operativo de seguridad y con la idea de negligencia, ya que para estos actores el espacio físico donde se llevó a cabo el recital no poseía las condiciones aptas para que se lleve a cabo. Rosa, una joven militante del PO integrante de la CVJ, expresó durante la movilización que: <<la música no mata. Lo que mata es la falta de previsión y organización. No queremos nunca más una muerte en un recital >>.

Question, Vol. 1, N. ${ }^{\circ}$ 65, abril 2020. ISSN 1669-6581

Instituto de Investigaciones en Comunicación | Facultad de Periodismo y Comunicación Social | Universidad Nacional de La Plata La Plata | Buenos Aires | Argentina

Página 8 de 13 
Los reclamos presentados por la CVJ se cristalizaron en la lectura de un documento público en el marco de la movilización en la plaza. Este documento fue previamente discutido y elaborado dentro de la Comisión. Sobre este proceso, Rosa comentó que:

\begin{abstract}
Empezamos a comunicarnos con más gente. Gente que empezó a escribir, que quería hacer algo, que veían la necesidad de organizarse... Las chicas de Salud, que con todo lo que había pasado están re calientes con el accionar del Municipio. Porque quedaron como muy comprometidas y de denunciar el abandono que hicieron con esos pibes. Convocamos a la primera reunión, cayeron un par de personas. Tuvimos varias reuniones más y ahí conformamos la Comisión (Registro de campo. Marzo de 2017).
\end{abstract}

En el discurso de Rosa se notaba gran preocupación para que la CVJ fuera considerada como un grupo heterogéneo. Dicha preocupación estaba fuertemente vinculada con la posibilidad de que el reclamo no se considerase una cuestión partidaria:

\begin{abstract}
Después del primer comunicado decidimos conformar una Comisión para que no sea una cuestión del partido, sino que se vea que hay vecinos, que hay estudiantes y demás que están participando. La idea es tomar eso como un reclamo transparente, porque si no lo que se hace es 'ah, no... esto es partidario, es la oposición' y no es así. Si bien somos un partido político, nos presentamos a elecciones con todo lo que eso implica, nuestra militancia no va por la chicana política, sino por lo que realmente creemos que es justo, por dónde están las responsabilidades y demás y denunciar eso. Lo mismo hicimos con las inundaciones de La Plata; lo mismo hicieron los compañeros con Cromañón (Registro de campo. Marzo de 2017).
\end{abstract}

En esa cita, resalta la importancia de la presentación de un reclamo transparente, que sería posible solamente si no se politizaban las demandas. Esta distinción que ella hace tiene el objetivo de "despegarse" de la posibilidad de que el reclamo sea considerado como una estrategia política, idea que opacaría lo verdaderamente justo. La preocupación objetivada por Rosa no es casual, debido a que el PO exterioriza una marcada oposición en la arena política con Cambiemos, alianza a la cual pertenece el intendente municipal y a quien eran dirigidos los cuestionamientos y las exigencias. Dicha confrontación tiene bases históricas, ya que el PO suele caracterizarse - en el desarrollo de su praxis política - por el esfuerzo de mantener una rivalidad declarada con todo sector considerado oficialista.

Esto lleva a pensar y reflexionar acerca de la idea de politización o no politización de un reclamo, asunto que ya ha sido abordado por Zenobi (2011):

[...] la categoría "la política" aparece con un carácter ambiguo: no se trata de una actividad valorada apriorísticamente de un modo negativo ni positivo, sino que al ser pensada como una actividad desarrollada por personas concretas, que están inmersas en un entramado de relaciones sociales al interior de movimiento y vinculadas a una

Question, Vol. 1, N. ${ }^{\circ} 65$, abril 2020. ISSN 1669-6581

Instituto de Investigaciones en Comunicación | Facultad de Periodismo y Comunicación Social | Universidad Nacional de La Plata La Plata | Buenos Aires | Argentina

Página 9 de 13 
historia en el mismo, la misma puede ser bien o mal practicada. De esta forma, hay familiares que actúan políticamente de un modo considerado como adecuado, otros son acusados de hacerlo de forma politizada. En este contexto, politización implica un tránsito entre formas diversas de hacer y de vincularse con la "política">> (p.121-122).

Como señala dicho autor, no se trata de si es o no una cuestión política sino cómo en el marco de una situación los actores consideran unas $u$ otras formas para vincularse con la política. Pero esto también permite visualizar cómo es que en la contienda los actores sociales constantemente elaboran y reelaboran las formas que ellos consideran más adecuadas para concretar las acciones públicas; acciones que, son motorizadas por sus propios intereses y que permiten concretar sus objetivos (Leach, 1976).

Ahora bien, ¿qué sentidos envuelve el uso situado de las categorías de verdad y justicia en el trabajo de la CVJ? A continuación, se intentará esbozar algunas respuestas.

\section{Verdad y justicia como tejido discursivo}

Las nociones de verdad y justicia, elegidas por la CVJ para acompañar sus consignas, conglomeran, en Argentina, un conjunto de sentidos vinculados al repertorio construido por diversos organismos, asociaciones y militantes por los Derechos Humanos para denunciar las vejaciones cometidas por el terrorismo de Estado durante la última dictadura cívico-militareclesiástica. De hecho, durante la lectura del documento presentado en la movilización, la CVJ invitó a participar a la comunidad olavarriense a las actividades de un acto que se llevaría a cabo el 24 de marzo por el Día Nacional de la Memoria por la Verdad y la Justicia en la ciudad. Da Silva Catela (2008) explica que ambas categorías, junto con la de memoria, lograron constituirse como un problema social gracias al trabajo político de los diversos organismos y agrupaciones por los DDHH. Como expone la autora, estas nociones se asocian a

[...] una matriz de interpretación que remite al terrorismo de Estado en la última dictadura y a las formas de hacer política y crear demandas de justicia inventadas y socializadas por las organizaciones nacidas durante la dictadura y ante la desaparición de personas. Así, el discurso de los derechos humanos y la memoria han triunfado como una poderosa bandera de acción moral y cívica (p. 12).

Da Silva Catela explica que hay muertes que producen indignación social. Frente a esto, los actores sociales recuperan una serie de símbolos y cuadros morales ya consagrados; se recurre a lo que <<se tiene a mano >>, a la estructura de significaciones que logra conectar lo que sucede con los Derechos Humanos y la memoria (Da Silva Catela, 2008). En este sentido, las nociones de verdad y justicia movilizan en Argentina un gran poder simbólico y que, en 
diferentes momentos y circunstancias, pueden ser manipuladas, usadas y resignificadas por actores sociales situados (Da Silva Catela, 2008). Por su parte, Fonseca y Cardarello (2004) explican, para el caso de Brasil, que la cuestión de los Derechos Humanos en su forma abstracta carece de significado. Sostienen que deben ser comprendidos más bien, desde su uso práctico y atendiendo a sus particulares consecuencias: dependen siempre de relaciones de poder producidas en contextos específicos <<expresadas en categorías semánticas precisas $>>$ (p. 8).

Entonces ¿Cómo son traducidas ambas categorías de alcance nacional en el reclamo particular que aquí se analiza? Ante las sospechas de ocultamiento de información por parte del Estado sobre la cantidad y causas de muertes durante el recital, la CVJ presenta la idea de verdad, conectando los sucesos con las muertes y desapariciones sucedidas durante la dictadura. En ambos casos, se coloca al Estado como el responsable de las situaciones indebidas, se lo pone en jaque, se lo cuestiona, y se le exige una resolución. A su vez, se actualiza el pedido de justicia, enlazando su accionar (el de la CVJ) con el activismo desarrollado por los familiares de las víctimas del terrorismo de Estado.

Este tejido discursivo le permitió a la CVJ llevar adelante un proceso de <<generalización de la denuncia > (Bolstanski, 2017), inscribiendo sus reclamos a un contexto más amplio y buscando el apoyo político de diversos actores políticos. Esto también da pistas para desentrañar el proceso de definición y certificación del carácter trágico de la situación analizada, ya que los actores sociales adhieren el caso a circunstancias que ya han sido acreditadas en la arena pública de esa manera. Nos muestra, además, que son categorías dinámicas, desde el punto de vista de los múltiples usos que los grupos y las personas realizan en función de sus propios intereses, estrategias, demandas y reclamos.

\section{Consideraciones finales}

A lo largo del artículo se buscó analizar cómo las categorías de verdad y justicia presentadas por las CVJ sirvieron como un tejido discursivo para acreditar el carácter trágico de las muertes sucedidas durante el recital de Indio Solari en Olavarría, así como dispositivos legítimos de reclamo hacia el Estado. Se mostró que ambas nociones aparecen asociadas a los repertorios producidos por diversas agrupaciones que luchan por los Derechos Humanos en Argentina, y como mecanismos de denuncia y reclamo por las muertes y desapariciones llevadas a cabo por el Estado durante la última dictadura militar. El proceso de apropiación de las nociones de verdad y justicia como las principales consignas de la CVJ, muestra el carácter dinámico de 
estas categorías y la importancia de comprender cómo son recuperadas, usadas, resignificadas por actores situados.

Por último, se quisiera destacar que esta miscibilidad entre dos hechos sociales (el recital y la dictadura militar) que en principio parecen ser insolubles entre sí por su distancia temporal y características singulares, admite comprender que los conflictos y controversias públicas no surgen ex nihilo, sino que se construyen a partir de la actualización de ciertas significaciones (históricas, sociales) que les dan forma, contenido y sentido.

\section{Agradecimientos}

A mi amiga y colega Natalia Larrea, por las lecturas y los aportes realizados para este artículo.

\section{Referencias bibliográficas}

Boltanski, L. (2017). Un nuevo régimen de justificación: la ciudad por proyecto. Revista de la Carrera de Sociología. 7 (7), 179-209.

Cefaï, D. (2012). ¿Qué es una arena pública? Algunas pautas para su análisis pragmático. Traducción ampliada de Nardicchione y Acevedo. En: Daniel Cefaï e Isaac Joseph, La herencia del pragmatismo. Conflictos de urbanidad y pruebas de civismo (pp. 51-81). La Tour d'Aigues, Editions de l'Aube.

Cefaï, D. (2014). Investigar los problemas públicos: con y más allá de Joseph Gusfield. En: Joseph Gusfield, La cultura de los problemas públicos, El mito del conductor alcoholizado versus la sociedad inocente (pp. 11-60). Cuidad Autónoma de Buenos Aires, Argentina: Siglo Veintiuno Editores.

Da Silva Catela, L. (2008). Derechos humanos y memoria. Historia y dilemas de una relación particular en Argentina. Juiz de Fora. 8 (1/2), 09-20.

Fonseca, C. y Cardarello, A. (2004). Derechos de los más y menos humanos. En: Sofia Tiscornia y María Victoria Pita (editores), Derechos humanos, tribunales y policías en Argentina y Brasil. Estudios de antropología jurídica (pp. 7-39). Ciudad Autónoma de Buenos Aires, Argentina: Colección de Antropología Social. Sección de Antropología Social.

Gluckman, M. (1958). Análisis de una situación social en el país zulú moderno. Manchester, Inglaterra: Manchester University Press.

Guizardi, M. L. (2012). Conflicto, equilibrio y cambio social en la obra de Max Gluckman.

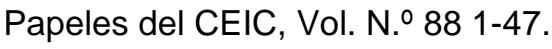

Question, Vol. 1, N. ${ }^{\circ} 65$, abril 2020. ISSN 1669-6581

Instituto de Investigaciones en Comunicación | Facultad de Periodismo y Comunicación Social | Universidad Nacional de La Plata 
Gusfield, J. (2014). La cultura de los problemas públicos. El mito del conductor alcoholizado versus la sociedad inocente. Cuidad Autónoma de Buenos Aires, Argentina: Siglo Veintiuno Editores.

Leach, E. (1976). Sistemas políticos de la Alta Birmania. Un estudio de la estructura social Kachin. Barcelona, España: Editorial Anagrama.

Pita, M. V. (2009). Las formas de la protesta. Violencia policial y familiares de gatillo fácil. En: Sofía Tiscornia, Roberto Kant de Lima y Lucía Eilbaum (organizadores), Burocracias Penales, administración institucional de conflictos y ciudadanía (pp. 83-118). Ciudad Autónoma de Buenos Aires, Argentina: Antropofagia.

Rockwell, E. (2009). La experiencia etnográfica. Historia y cultura en los procesos educativos. Ciudad Autónoma de Buenos Aires, Argentina: Paidós Editorial.

Zenobi, D. (2011). La politización del movimiento Cromañón entre los "modelos caseros" y los "modelos del observador". Revista Avá, 21, 107-125. 